\title{
IMPROVED PART MODELING IV A PROCESS PLANNING SYSTEM
}

\section{Ali CALISSKAN \\ Bahadir TANTAY \\ Filiz YAĞCI \\ Agean University Faculty of Science Department of Math.}

\begin{abstract}
An expert process planning and fixturing system described in [14] is improved here by applying Boolean operations. This system uses forward planning strategy, i.e. begins with the blank part and applies some operations for each face or feature until reaching the finished part. This paper is focused on finished and intermediate part modeling within the process planning system by geometric transformations and Boolean operations.
\end{abstract}

\section{INTRODUCTION}

The Product Data Exchange Specification (PDES) has been designed by the IGES/PDES Organization. This new standard has been developed under the name of Standard for the Exchange of Product Model Data (STEP) which is a neutral graphics standard capable of representing product data completely and comprises both geometric and technological information including geometry, topology, form, features, tolerances, surface roughness and material necessary to define a component completely, [2,3]. Consequently, through the use of STEP, data transfer between dissimilar CAD (Computer Aided Design) systems and between $\mathrm{CAD}$ and CAPP/CAM (Computer Aided Process Planning/ Computer Aided Manufacturing) systems can be achieved, [4].

CAPP is an important activity which bridges CAD to CAM and translates the design information into manufacturing instructions to produce mechanical components, $[5,6,7,8]$. A flexible, automated, generative CAPP system using expert system techniques and the STEP standard, called EPPSU, has been developed, [9]. The system is implemented in Prolog and runs under UNIX. The information about a blank part and material is an input to the process planning system. The system developed utilizes forward planning. When the system developed receives 3D B-rep CAD data for prismatic parts in STEP format, it converts such data format into Prolog clauses form, designs a blank for the part and selects a stock size. First, the features in the component are identified and 
evaluated.Having calculated the angles between faces, found neighbouring relationships of the faces, the blank and finished part models are represented in a special format. Besides selection of a reference surface, operation selection for each feature, calculations of machining parameters, selection of cutters and machine tools are carried out. Fixturing for each stage of operation is also addressed,[14].

In this paper we have also given a Pascal implementation of this method. In this implementation, the image of the part is got by using rotations and projections.

\section{FINISHED PART MODELING}

Before the representation of the finished part, some necessary information needs to be provided. After extracting the face direction, the neighbouring faces for each face are found and declared in the data base. Through checking common edges, the angles between faces are then calculated, followed by determination of convexity-concavity relationship between faces. The part is finally represented in a special format and written to the final model file including face and subfeature representation. Since two adjacent faces share a common edge, finding the common edges of the faces the neighbours of each face are declared in the data base.

For a complete image of the part, we must have coordinates of the vertices, connectivity matrice and outward normal vectors of the faces.

To check the visibility of the faces, first we must be sure that the normal is outward. To do this, we take a vector I directed inside the object, then find the normal vector $\mathbf{n}$ of the face.

If $\mathbf{n . I} \leq 0$, the normal is pointing outward.

After determining that the normal is outward, we construct a view vector $\mathbf{V}$ from any point of the surface to the viewpoint.

If $\mathbf{V} \cdot \mathbf{n}>0$, then the face is visible

In part modeling, images of the part from different points of view are needed. This is managed by projecting the part into a plane. There are 2 main types of Planar projections: Parallel and perspective. In parallel projections, the center of projection is located at infinity, so all projectors are parallel to each other; But for perspective projections, the center of projection is at a finite distance from the projection plane. Parallel projections can save the dimensions, but perspective 
projections give a more realistic image of the part. To project an inclined surface,

1. We find vectors $V_{1}$ and $V_{2}$ on the surface.

2. $\mathbf{V}_{1} \times \mathbf{V}_{2}=\mathbf{n}$, gives surface normal.

3. We rotate the normal, $\mathbf{n}$, until coinciding it with one of the coordinate axes.

4. We take the projection onto the plane perpendicular to the normal,[13].

\subsection{Calculation of Angles Between Faces}

In a STEP file, each face is oriented by a unit direction vector. The calculation of the angle between two faces is based on the angle between their direction vectors. The angle, $\theta$, between two unit vectors, $u_{1}$ and $u_{2}$ satisfies the following equation,

$$
\mathbf{u}_{1} \cdot \mathbf{u}_{2}=\left|\mathbf{u}_{1}\right|\left|\mathbf{u}_{2}\right| \cos \theta
$$

where $\left|u_{1}\right|$ and $\left|u_{2}\right|$ are the magnitudes of the vectors $u_{1}$ and $\mathbf{u}_{2}$, respectively.

\subsection{Processing of Features}

In a STEP file, the features such as steps, slots, holes, etc. are formed by transformations like sweeping and rotating. To do these, we can use Boolean operations like difference, intersection and union. Therefore, some more information for each feature namely the location and orientation of faces of the feature, the effect of the feature on other faces, etc. needs to be extracted. For sweeping along a straight line starting from the origin,

$T(s)=\left[\begin{array}{cccc}1 & 0 & 0 & 0 \\ 0 & 1 & 0 & 0 \\ 0 & 0 & 1 & 0 \\ a t & b t & c t & 1\end{array}\right] \quad, 0 \leq \mathbf{t} \leq 1$, sweeping matrice can be used. If there are also rotations along the path, rotation angles should also be considered. To rotate or translate closed polygons, we control the direction of the normal to the polygon. A common approach is to place the normal in the direction of the instantaneous tangent to the path,[13]

\subsubsection{Step Feature Processing}

A step feature sweeping profile, which is an " $L$ " is located and swept to form a step as shown in Figure 1. 


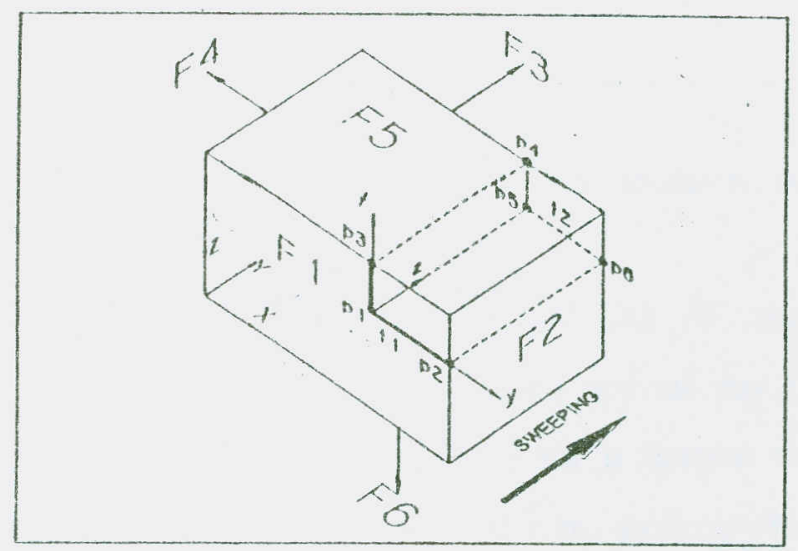

ligure 1. Lexation, orientation and sweeping an " 1, profile to form a step

The local coordinate system, the origin of the " $L$ " profile (P1), the distance between P1 and P3 and the sweeping length are explicitly defined in the STEP file. On the other hand the other points (P2,P3,P4,P5,P6), t1 and $\mathbf{t} 2$ distances need to be calculated. 11 and $\mathbf{t} 2$ distances are calculated as seen below. The intersection between a line and a plane can be defined by the following equation:

$$
a+u b+v c=d+t e
$$

This equation can be used to solve for $\mathrm{t}$ as

$$
t=\frac{(b \times c) \cdot a-(b \times c) \cdot d}{(b \times c) \cdot e}
$$

So, the coordinates of the points P2,P3,P4 and P6 (Figure 1) can be calculated.

\subsubsection{Slot Feature Processing}

Like a step feature, a slot feature is similarly formed by sweeping a " $U$ " shape (Figure 2). The width and height of the slot, the sweeping length and the local coordinate system with the coordinates of the origin, P1 are available explicitly in the STEP file.

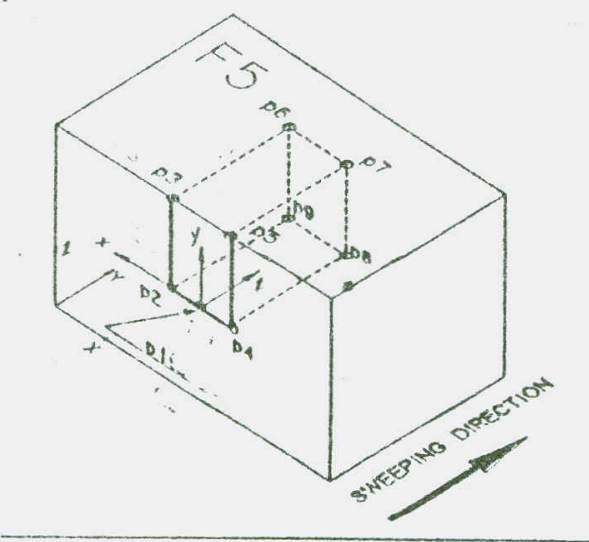

ligure 2. Sweeping a "U" shape to form a slot

\section{INTERMEDIATE PART GENERATION}

For fixturing purposes, to define the status of workpiece accurately, an 
intermediate part model for each set up is necessary. The selection of supporting, locating and clamping faces can be achieved on the basis of the intermediate model $[10,11]$. The blank, finished and each intermediate part model is represented in a special format and stored within the data base. The geometric model of each intermediate part is generated based on the operation performed to machine the feature.Each operation changes the geometry of the component. After performing each operation, the part model is updated with respect to the operation type.A new intermediate model must be represented in the data base before starting the fixturing procedure

The blank part model is eventually transformed into the finished part model. During this transformation, intermediate models are produced in relation to the operations performed. By examining the new intermediate model, the fixturing is accomplished for the next operation. When the fixturing procedure is accomplished for the operation to identify the supporting, locating and clamping faces, it is necessary to know enough information about the faces of the component, since location and clamping are achieved on basis of the basis of the faces. Thus, the information about the faces should be readily available within the data base. Not only the face information of the blank and finished part model but also the faces of every intermediate model should be stored explicitly in the data base. A special representation scheme has been developed to represent workpieces based on the faces and the neighbouring relationships of each face with others.

When an operation is applied to the part, the material is removed from the component with respect to the operation type and the model is updated. Such dynamic modification of the part model enables the fixturing program to consider the new generated faces for the next operation. Each operation affects the faces in different way. The procedure for generation of new intermediate models is based on the operation type and is outlined as follows:

$\square$ determine the coordinates of the new points to be generated by the operation,

$\square$ locate the new faces in the part model,

$\square$ update each face of the part model affected by the operation.

During the modeling of components before locating the necessary points in the component it is examined to determine if a point is on a line or on a 
plane. In some cases the intersection of two lines is used to locate a point in the part. If we want to use Boolean operations, we must create a Constructive Solid Geometry (CSG) table for the finished part. Let's examine the part below:

0.24

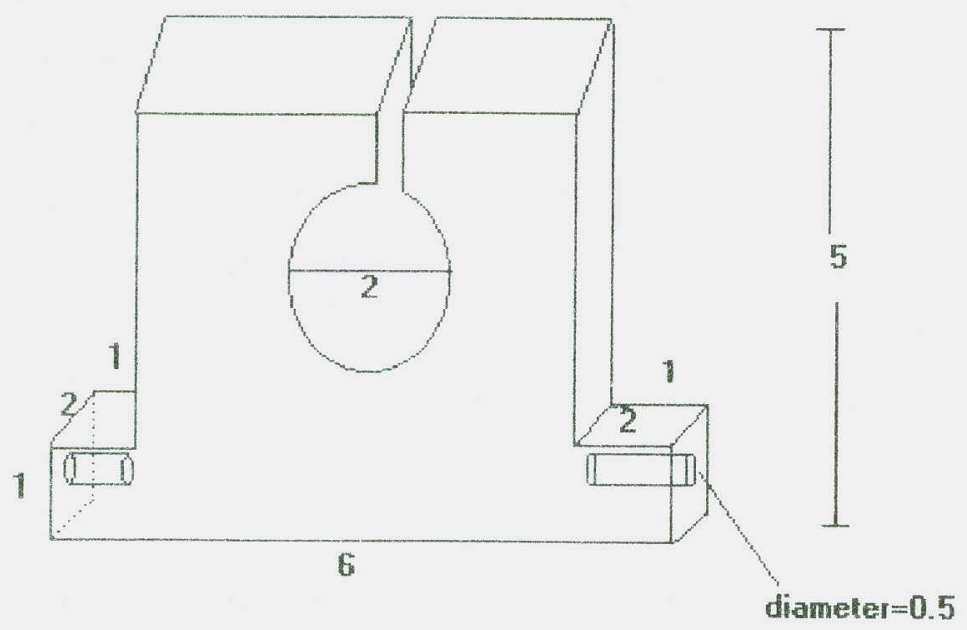

To model this part the following CSG table can be constructed,

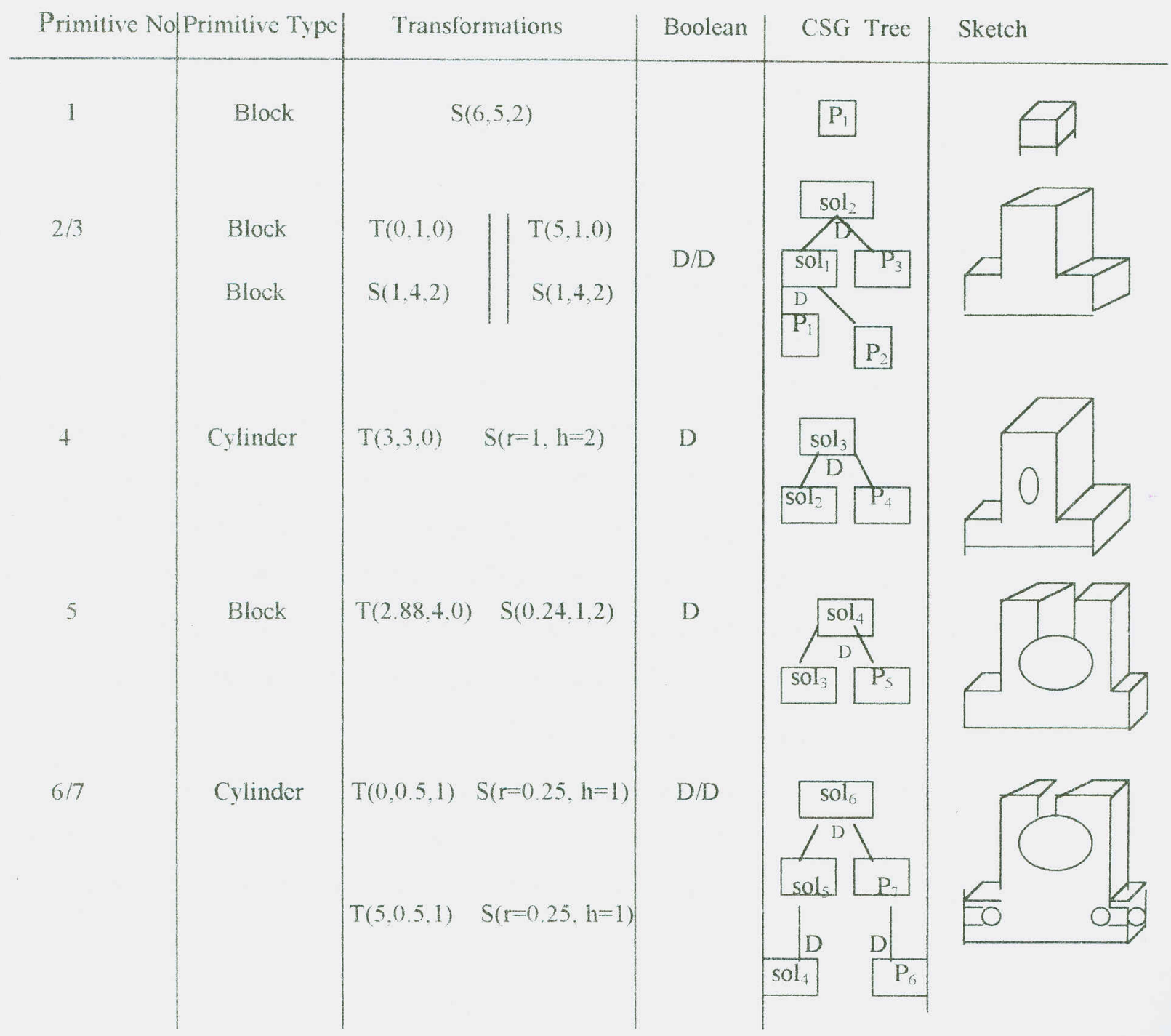




\section{IMPLEMENTATION:}

The main part of the Pascal implementation of this Part modeling is given below,

\section{BEGIN}

Grafik_Ekrani_Olustur;

SetBkColor(11);

NormVideo;

Olcekle(CekXY,CokXy);

OR_Otl(CokXY,OtIXY);

Oblique(OtXY, $+60,70$, ObLXY);

Prizmayi_Ciz(Ob1XY,9,20);

Parcayi_Karar_Ver_Cikar;

CloseGraph;

END.

\section{CONCLUSIONS:}

Finished and intermediate part modeling in a process planning system discussed in [14] is improved here. We apply Boolean operations to the part until getting the finished part model. In each step, the intermediate models showed in CSG table are defined in the data base. This is an active system and allows to choose the best operation type for each intermediate model until reaching the finished part model.

\section{REFERENCES}

1. STEP - Standard for the Exchange of Product Model Data, The First

Working Draft of STEP Version 1.0, ISO TC184/SC4/WG1, pp. 1-546, 1988.

2. Smith, B.M., "Product Data Exchange:The PDES Project-Status and

Objectives", US Department of Commerce, NIST - National Institute of Standards and Technology, NISTIR 89-4165, p.3, 1989.

3. Dong, X., and Wozny, M.J., "Managing Feature Type Dependency in a Feature Based Modeling System", Proceedings of ASME International

Computers in Engineering Conference Boston, Vol. 1,pp, 125-130, 1990.

4. Nolen,J., "Computer Automated Process Planning for World-Class

Manufacturing", James Nole \& Company, USA, 1989. 
5. Desai, V.S and Pande, S.S., "GFM -An Interactive Feature Modeller for CAPP or Rotational Components", Computer Aided Engineering Journal, pp. 217-221, 1991

6. Joshi,S and Chang, T., "Feature Extraction and Feature Based Design Approaches in the Development of Design Interface for Process Planning", Journal of Intelligent Manufacturing, London, Vol.1, No.1,pp.1-15,1990.

7. Cho,K.K.,Lee,S.H. and Ahn,J.H., "Development of Integrated Process Planning and Monitoring System for Turning Operation", Annals of the CIRP, Vol.40/1, pp. $423-427,1991$

8. Alting, L and Zhang, H., "Computer Aided Process Planning: the state-of-theart Survey",International Journal of Production Research,Vol. 27, No.4, pp. $553-585,1989$.

9. Gülesin, M., Jones, R.M. and Blount, G.N., "An Intelligent Knowledge Based Process Planning and Fixturing System Using Step Standard", Proceedings of The Third International Conference on Computer Aided Design, Manufacture and Operation in the Railway and Other Advanced Mass Transit Systems, vol 1, pp. 280-291 Washington DC, USA August 1992

10. Dong,X., and Devries, W.R. and Wozny, M.J., "Feature Based Reasoning in Fixture Design", Annals of the CIRP,Vol.40/1, pp.111-114, 1991.

11. Giusti, F., Santochi, M. and Dini, G, "KAPLAN: A Knowledge Based Aproach to Process Planning of Rotational Parts", Annals of the CIRP, Vol.38/1, pp $481-484,1989$

12. Mortenson,ME., "An Introduction to the Mathematics and Geometry, Computer Graphics", Industrial Press Inc., USA, 1989

13. Anand, V.B. "Computer Graphics and Geometric Modeling for Engineers", John Wiley \& Sons, Inc.

14. Çalışkan,A., Gülesin,M., and Tantay,B., "Part Modeling In A Process Planning System", Proceedings of the First National Econometry and Statistics Symposium İzmir, Vol.1, pp. 263-279, 1993 